\title{
A Precision Post-Operative Wellness Monitoring Solution
}

\author{
Nilmini Wickramasinghe \\ Epworth HealthCare and \\ Swinburne University \\ Australia \\ nilmini.work@gmail.com
}

\author{
Vijay Gehlot \\ Elliot B. Sloane \\ Dept of CS and CEET \\ Villanova University \\ USA \\ vijay.gehlot@villanova.edu, \\ elliot.sloane@villanova.edu
}

\author{
Phil Smart \\ Epworth HealthCare and \\ The Austin \\ Australia \\ philipsmart@easterncolorectal. \\ com
}

\author{
Jonathan Schaffer \\ The Cleveland \\ Clinic \\ USA \\ schaffj@ccf.org
}

\begin{abstract}
Multiple orthogonal challenges around escalating costs and providing quality care plague healthcare delivery, especially in OECD countries. This research in progress paper addresses the post-operative discharge phase of the patient journey and proffers a technology enabled model that both supports a quality care experience post discharge but also prudent management to minimize costly unplanned readmissions and thereby subscribe to a value-based care paradigm. The chosen context is stoma patients but the solution can be easily generalized to other contexts. Next steps include the conducting of clinical trials to establish proof of concept, validity and usability.
\end{abstract}

\section{Introduction}

Given the challenges facing private healthcare today and moving forward there is increasing pressure on private healthcare organizations to provide high value, high quality patient-centered care across the acute-care continuum (Australian Commission on Safety and Quality in Healthcare 2010, [1]). While the recognition for the need of care delivery to be patient-centric is growing, the appropriateness of the approaches adopted to achieving this endeavor remain questionable [2].

An integral enabler is without question Information Technology (IT) solutions (see for example $[3,4,5]$ ). The limitation with many current systems is their limited coverage across the acute-care continuum [6,7], where both pre-admission and post-discharge phases are not seamlessly connected to the hospitalization phase in the patient journey.

In Australia, as in other OECD countries like the US, unplanned readmissions are now being more carefully scrutinized. In most instances in Australia, unplanned readmissions are not reimbursed. i.e., readmissions within 28 days of discharge are considered to be related to the primary diagnosis/treatment, so the added cost must be borne by the provider (AIHW 2017, [8]). We believe it is possible to reduce the number of unplanned readmission by developing precision post discharge wellness monitoring solutions, and the following serves to outline this solution and answer the research question: "How can we design a suitable technology solution to support post discharge monitoring?"

We select stoma patients as a pilot study for this solution because we note that based on hospital data gathered from a large not-for-profit tertiary institute in Melbourne, Australia a common and avoidable unplanned readmission relating to stoma patients is due to inadequate hydration. This is particularly problematic during the hot dry Australian summer months.

This paper proposes a generic, open-source-based starting point for a customizable modeling, simulation and testing framework for mobile Patient Care Devices (PCDs) that can support relatively complex coordination of care for post-surgical patients. This system uses an architecture that can be modified to suit each patient's precise needs or widely differing clinical protocols. These tools allow simulation of expected ranges of safe and reliable performance, and can also be used to explore or simulate likely failure modes and potential safety or health risks due to communication system or staffing overload, errors, or other complications.

\section{Background}

In a recent study by the International Surgical Group on the dynamics of surgery across the globe, the findings indicated that over 320 million people globally have undergone surgical procedures; approximately $17 \%$ of these patients develop several complications and 
among them nearly $2.8 \%$ pass away due to these complications. It can, therefore, be estimated that approximately 1.5 million patients annually or three patients per day die due to post-operative complications. For instance, Bartels et al (2013) argue that in America, if the postoperative mortality was to be included in the official statistics as per the data from the Centers for Disease Control and Prevention, it would depict the third leading cause of death after heart illness and cancer [15]. Succinctly, many patients die in the ward or post discharge, where the doctor to patient ratio is low and where the individuals are not continuously monitored. Monitoring patients beyond the operating room and ICU may enable early detection of medical deterioration and timely interventions (Michard et al., 2015, [16]). Michard et al. (2015) notes that postoperative complications are not only a human burden but also a dramatic increase in the hospital expenses. As a result of this clinical and economic burden, a number of initiatives have been developed to offer an advanced quality of surgical care, including clear surgical safety specifications, minimally-invasive surgical procedures, protecting mechanical ventilation, and improved postsurgical recovery initiatives (Bartels et al., 2013, [15]). Due to recent technological advancements and innovations in healthcare systems it is becoming easier to monitor patients in the ward and post discharge and this in turn serves to improve patient satisfaction and overall outcomes.

"Prehabilitation" is one of the elements that are thought to have a great influence on the postoperative outcome. Ideally, preoperative improvements in the physical status in addition to better control of risk factors can be enhanced by digital tools and smart applications which are downloaded on smartphones and in tablets (Michard et al., 2017, [17]). According to Michard et al. (2017), the connected devices including brachial cuffs as well as electronic scales are often used for self-monitoring of blood pressure and weight, better control of hypertension and weight gain, and the visualization of the surgical trends over time.

However, a growing area of focus is now on post discharge and rehabilitation monitoring. Numerous sensors and monitoring systems are being designed and developed for proactive post operated patients as well as ambulatory patients. According to Michard et al. (2017), smart software has been developed to prevent alarm fatigue, to filter artifacts, and to fuse vital signs into the postoperative wellness indexes or the warning scores that are used for easy and visual identification of health deterioration, or even the prediction of hostile events beforehand (Pinsky et al., 2016, [18]).

Intraoperative fluid monitoring is one of the major determinants of postoperative outcome (Pinsky et al. 2016). Over the years, fluid overload has been known to be a major factor responsible for complications that are related to tissue oeadema such as prolonged mechanical ventilation along with anastomotic leak (ibid). Thus, fluid restrictions have been encouraged to some extent. Nevertheless, recent researches such as the one by Thacker et al (2016) have clearly illustrated that insufficient fluid management is linked to a considerable increase in postoperative complications [19]. According to Michard et al. (2017), titrating and modifying fluid management to patient's needs is highly desirable to make certain that patients receive the correct amount of fluid at the appropriate time. Multiple noninvasive hemodynamic monitoring interventions are currently available, including bio-impedance tracheal tubes, volume clamp approaches, application tonometry and bio-reactance surface electrodes [17]. These offer physicians an opportunity to measure and track any change in the blood flow during the therapeutic programs and to rationalize fluid management. Benes et al. (2014) state that precluding unjustified fluid management by detecting fluid unresponsiveness has been considered to be important to minimize postoperative morbidity, hospitalization time, and cost [20]. Some of the noninvasive parameters, including pulse pressure variation from volume clamp approaches and $\mathrm{SpO} 2$ variability index from the pulse oximeters, are helpful in detecting fluid unresponsiveness [20].

Pulse oximeters were used to continuously monitor the $\mathrm{SpO} 2$ as well as heart rate among 2,841 orthopedic patients. According to Taenzer et al. (2010), this use was linked to a significant decline in the number of rescue events and ICU transfers. Piezoelectric contact-free sensors placed under the mattress aids in the continuous monitoring of heart rate and respiratory rate among 2,314 medico-surgical patients [21]. This patients' population has been linked to a significant decrease in the number of calls for hospitalization and cardiac arrest (Brown et al., 2014, [22]).

Subbe et al. (2017) contend that wireless sensors have been invented recently to help in monitoring vital signs such as the heart rate, respiratory rate, $\mathrm{SpO} 2$, and blood pressure. Such solutions automatically calculate an early warning score, and then alert nurses if deterioration has been detected [23]. This can be associated with a considerable decrease in the number of cardiac arrests and mortality rates.

There has been a strong correlation between intraoperative hypotension and postoperative difficulties such as acute kidney injury, stroke, and myocardial injury. It has been established that intermittent blood pressure measurements fail capture every patient's hypotension events in a timely manner (Chen et al., 2012, [24]). According to Chen et al (2012) clinicians may miss nearly seven minutes of 
hypertension per hour during a three hour orthopedic or abdominal procedures, especially when using the intermittent blood pressure measurement. Recently, it has been suggested that only a minimal number of minutes of hypertension can significantly affect the postoperative outcome. Thus, though fatality from intraoperative hypotension and postoperative difficulties has not yet been identified, it appears desirable hypotension events as much possible (Salmasi et al. 2017, [25]). This may need a more rational as well as controlled use of the anesthetic agents, especially during induction. It may also require continuous monitoring of blood pressure with the non-invasive methods for the immediate detection and correction of any considerable blood pressure drop.

Taken together, we can see there is a trend and potential to embrace technology solutions for postoperative monitoring; however, what is key is to have focused solutions. This paper tries to address this and identify critical issues for the design and development of post discharge monitoring of stoma patients.

\section{Clinical Use Case}

In order to provide proactive patient care postdischarge following stoma surgery, several remote medical devices can be employed. For example, elevated patient temperature or pulse can indicate an emerging infection at home which might easily and inexpensively be treated by early intervention with an appropriate antibiotic. High blood pressure and pulse might be an indicator of patient pain or discomfort, which might be initially treated with basic over-thecounter anti-inflammatory medications. Low blood pressure and elevated pulse might be predictors of dehydration, which might readily be treated by drinking more fluids and electrolytes. The stoma bag may also be monitored with simple sensors that keep track of filling and emptying rates, providing an indication of inadequate food and liquid intake.

In all cases, the monitored patient wellness parameters can be routed to a central patient homecare coordination team. The care coordination team can invoke appropriate rules and treatment actions. For example, a care coordinator could call the patient/ family, provide remediation guidance, education, and/or prescriptions. If needed, a visiting nurse or physician could be dispatched to provide in-home care.

In addition, the monitors and the data monitoring systems can have alarm and/or alert level triggers preset or remotely adjusted. Thus, if a low grade fever appears to be emerging, the patient, family, or care coordination team could increase the temperature alarm by one degree, to notify them if/when the fever becomes more severe. Similarly, a high and low blood pressure alarm could be pre-established based on the patient's discharge condition, in order to alert caregivers of unusual emerging risks.

Once a patient's physiological data is available to the care coordination system and team, clinical decision support algorithms and systems can be used to enhance, accelerate, and escalate emerging patient risks to staff who are appropriately trained to support high-quality, safe home care for discharged patients. Intervention at the home will usually be far, far less expensive than readmission to the hospital, and care plan changes can often occur quickly. The care coordination team will be able to arrange emergency care or transport if home-care turns out to be inadequate for a specific patient situation. Because many physiologic monitoring devices are now becoming rather inexpensive and ubiquitous, and they rely on consumer-grade internet or cellular communication channels, the incremental cost of deploying such systems is falling rapidly. In addition, many of these devices can be cleaned and re-used for subsequent patients.

In the following sections, we illustrate the system design and simulation of a flexible home-care monitoring and care coordination system. We identify representative monitoring devices, but the model is extensible. Additional monitoring devices and data can easily be added. e.g., in some cases patient weight may be a valuable indicator of dehydration, or of congestive heart failure complications. Adding a patient scale and decision support rules is very easy with this system.

In addition, this system could easily be extended to include patient co-morbidities. For example, a severe industrial or automobile trauma patient could conceivably be sent home with both a stoma bag and a hip replacement. If that were the situation, additional physiologic channels may be added, such as gait and PT/exercise/mobility tracking and analysis.

\section{Modeling Approach and Method}

Our focus is to create a simulation model based flexible framework to assess and validate various protocols and workflows for post-operative and postdischarge care. Towards this end, we are using the Colored Petri Nets (CPN) modeling and simulation approach $[9,10,11]$.

CPNs are extensions of the widely studied Petri Net formalism, which is a graphical notation for modeling systems [12]. CPNs combine the graphical components of Petri Nets with the strengths of a high level programming language, making them suitable for modeling complex systems. Petri Nets provide the foundation for modeling concurrency, communication, 
synchronization and resource sharing constraints, while a high level programming language provides the foundation for the definition of data types and the manipulations of data values. As we show later on, support for compound data types is crucial for representing and processing different data details of a variety of monitoring devices. The CPN language allows the model to be represented as a set of modules, allowing complex nets (and systems) to be represented in a hierarchical manner.

CPNs allow for the creation of both timed and untimed models. Simulations of untimed models are usually used to validate the logical correctness of a system, while simulations of timed models are used to evaluate the performance of a system. Timed activities play an important role in home-care monitoring and care coordination systems.

\section{Simulation Model}

As mentioned above, we use CPNs to create a flexible framework for postoperative wellness monitoring. In particular, we use the hierarchical approach of the CPNs. Figure 1 depicts the top level system view of this model.

In Figure 1 we have also explicitly identified care coordination and vigilance as separate layers: "Community Care Coordination Hub" and "Home Data and Alerting Aggregation System". These layers abstract away clinical vigilance alerting rules that are needed for various clinical care coordination categories, allowing customization to suit the situation. For example, systolic and diastolic high blood pressure alert limits might trigger when any patient's BP exceeds either $180 \mathrm{mmHg}$ systolic or $140 \mathrm{mmHg}$ diastolic, but individual patient overrides are likely needed. There should be care coordination rules to set personal limits higher or lower based on medication and clinical situation. Automatic implementation rules may also require the ability to fetch relevant data from an EHR.

Each of the six rectangles with double border in Figure 1 are called Substitution Transitions. Each of these Substitution Transitions represents a distinct page/layer/module/subsystem that becomes integrated into the entire model. For the purposes of this paper, we will use the term Submodule net to refer to these Substitution Transitions. In essence, each Submodule net can be evolved into a complex CPN subsystem if/as needed, facilitating modular design and testing. In each referenced Submodule net, we can assemble all needed details to support the underlying activity or sub-system.

In defining this framework, we have chosen a general scenario where patients condition is monitored by a variety of devices. This is consistent with the basic architecture used in IHE International's Patient Care Device Domain (IHE-PCD) standards (www.IHE.net, and the HITSP IS-77 and TN-905 at www.HITSP.org). Each PCD (device) functions like a stand-alone IoT device. In the HITSP documents above, two architectures are offered: the discrete device model and the local patient data aggregation hub model that is embodied in the Continua and the ICE/OpenICE (Integrated Health Environment) standard. In this second architecture, a home data aggregation hub stores data until it is polled (store-and-forward). We model the second approach. Thus, as shown in Figure 1, the data from these devices is sent to a Home Data and Alerting Aggregation System via the underlying Home Network.

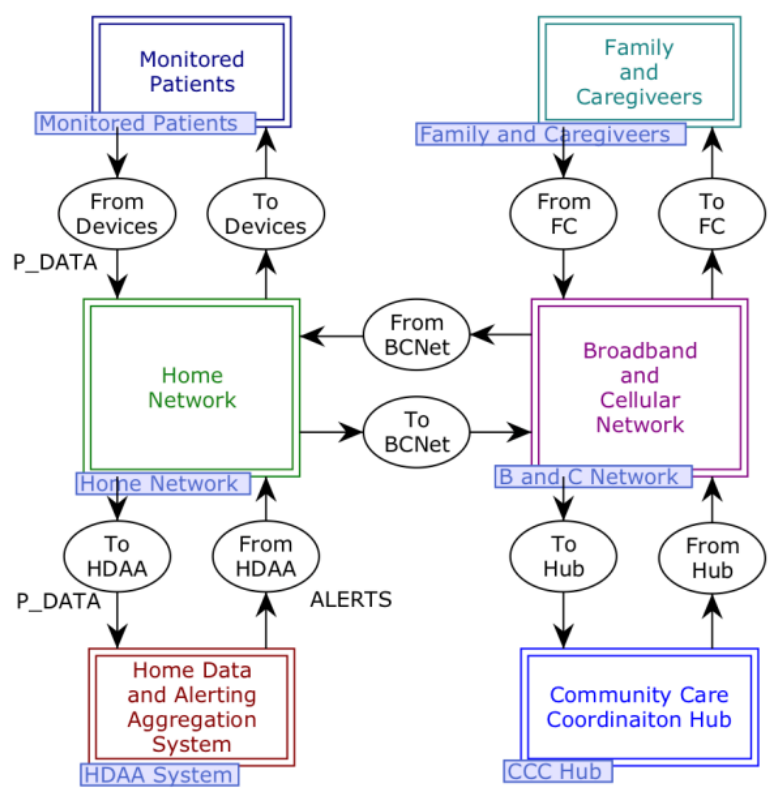

Figure 1: CPN model of our framework for post discharge care

The component Monitored Patients consists of three categories of PCD monitors, sensors, and/or apps: Wearable Sensors, Homecare Sensors/Apps, and Mobile Self-Reporting Apps. Figure 2 shows the subcomponent associated with Wearable Sensors.

Each category allows any number of patients and devices. This is achieved by modeling each device behavior as a generic net and then populating that net with unique patient IDs and unique device IDs. For example, Figure 3, below, shows the net associated with a blood pressure monitoring device. 


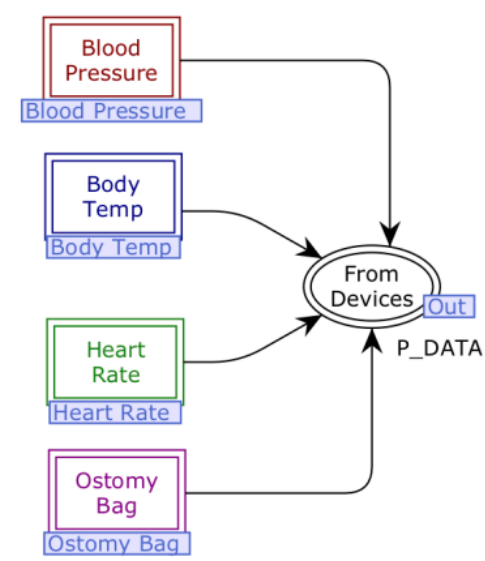

Figure 2: Wearable Sensors model component depicting various sensors that may be worn by a patient



Figure 3: The net describing the behavior of a blood pressure monitoring device

As mentioned before, the single CPN submodule net that is shown in Figure 3 can keep track of any number of patient-device pairs. We achieve this by defining a color set (i.e., a data type) consisting of a PatientID and a DeviceID. Assuming we have an stoma patient [PatientID_1] wearing a blood pressure monitoring device [DeviceID_1], and an ortho patient [PatientID_2] wearing a different blood pressure monitoring device [DeviceID_2], the initial marking 1 (1,2)@5+++1 (2,1)@10 depicted on place Patient Data Trigger will initialize the net with two timed tokens. The first one will trigger a reading at simulation time 5 and the second one will trigger a reading at time 10. The depicted function bpTrigger(pd) is a customizable function that sets the trigger for the next reading. The current blood pressure reading is sent by the firing of the transition Send BP Data and the associated data is generated by $\operatorname{bpData}(p d)$. For example, the current token depicted in green residing in place From Devices shows that a reading for PatientID_1 of 195/62 was generated at time 143 by DeviceID_1. This data packet will be sent over the home network to Home Data and Alerting Aggregation
System. As mentioned before, this system is responsible for collecting and storing data locally as part of a patient's Personal Health/Medical Record (PHR/PMR). The stored data may be polled remotely by a Community Care Coordination Hub or even by Family and Caregivers over a broadband network as shown in Figure 1. The aggregator/hub can itself add vigilance, alerts, and other functionality depending on the underlying protocol for patient monitoring and care. Figure 4 shows the subnet associated with the Home Data and Alerting Aggregation System in our CPN model.

In this net, the place PMR collects and aggregates patient data. The current token shown in green, depicts the various device data that has been collected for each of the two patients. For example, the entry $(4,25, \operatorname{TEMP}(97))$ means at time 25 , a temperature reading of 97 was recorded for PatientID_1 and the reading was generated by DeviceID_4. Similarly, the

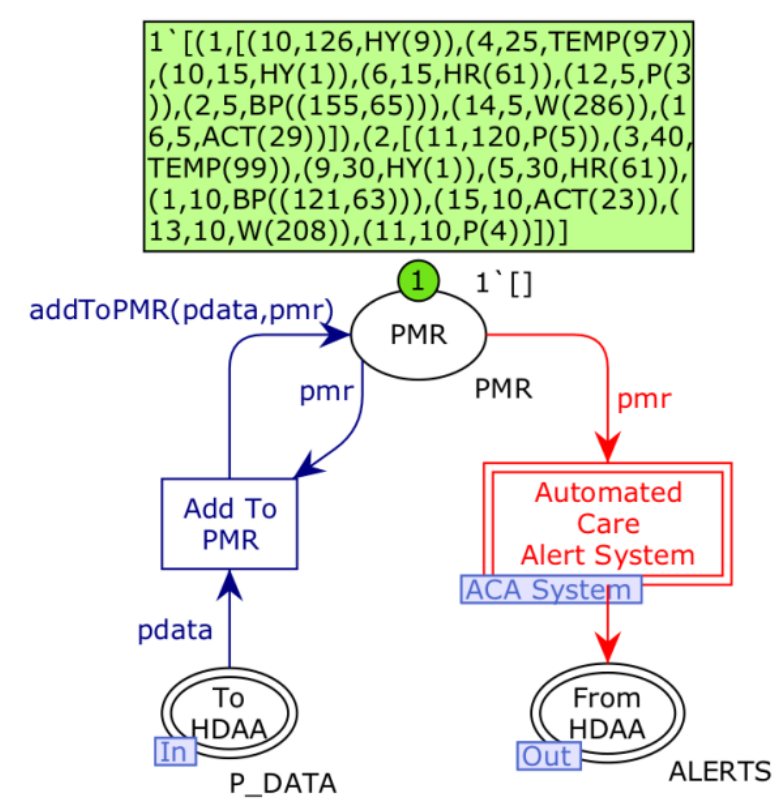

Figure 4: Patient device data aggregator and alerting system

entry $(11,120, P(5))$ means at time 120 PatientID_2 reported a pain level of 5 via a Selfreporting_appID_11.

Figure 5 below shows the subnet associated with an ostomy bag that has a "bag full" alarm built into it. Since only patient 1 is a stoma patient, the net is initialized only with patient ID 1 . Thus, our framework and model allows flexibility both in terms of number of devices as well as type of devices associated with a patient to be monitored.

The net monitors the current level and generates an "ostomy bag full" alarm if the level reaches a defined 
alert level via the firing of the transition Send OBag Alert. The level is checked and alert is generated by function $\operatorname{bagAlarm}(p d, l)$. The current green token in place OBag Level shows the current level to be 3 .

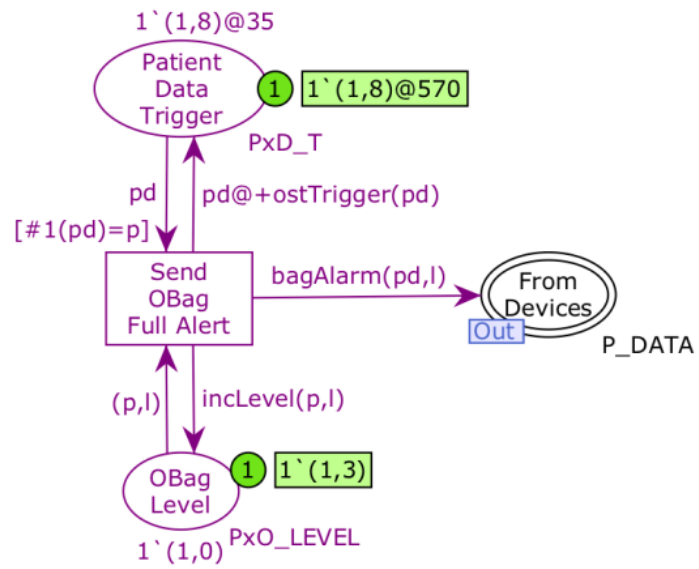

Figure 5: Net associated with ostomy bag

The CPN Tool models provide an important additional opportunity: when ready for clinical testing, each Sublayer net may be replaced with functional programs and/or real devices and sensors can be interfaced to feed actual patient data directly into the model. That allows incremental conversion of the CPN model from a simulation to a functional clinical tool. This facilitates straight-forward pilot testing, functional validation, and full system scalability verification prior to widespread clinical deployment. In addition, different clinical decision support systems and rules can be substituted without disturbing the rest of the system, enabling customization and research to match evolving needs.

\section{Results and Discussions}

The previous section proffers a solution that leverages the capabilities of various technologies. The aim is to monitor patients as unobtrusively as possible so that alerts can be triggered to inform the designated healthcare professional if a trigger incident has occurred that should be addressed. In this way, the patient is able to navigate the post-discharge phase of their treatment effectively and efficiently, with the highest level of positive patient and caregiver experience. In addition, by having alerts promptly triggered if a problem arises it is also possible to act as quickly as possible, thereby averting a more complicated, dangerous, and/or expensive problem further down the track. In this way, we believe we are addressing two critical objectives of healthcare delivery simultaneously; namely providing a high quality patient experience as well as providing a high value solution as we are trying to mitigate the need for unplanned readmissions by catching trigger situations as early as possible and then addressing them.

The next step is to run a two arm non-blinded clinical trial to test the full benefits of the proposed solution. The control arm will continue to have patients exposed to standard practices around discharge and post discharge follow up and monitoring while the intervention arm will focus on using the developed technology solution in addition to standard care practices around discharge and post-discharge management. In particular we plan to monitor levels of hydration, using triggers of blood pressure, pulse and weight to trigger levels going below an appropriate threshold. In addition, patients will receive via their mobile phones education, reminders and other important information about maintaining appropriate levels of hydration. We focus on hydration as this has been identified as the singular most frequent reason for unplanned readmission with stoma patients at

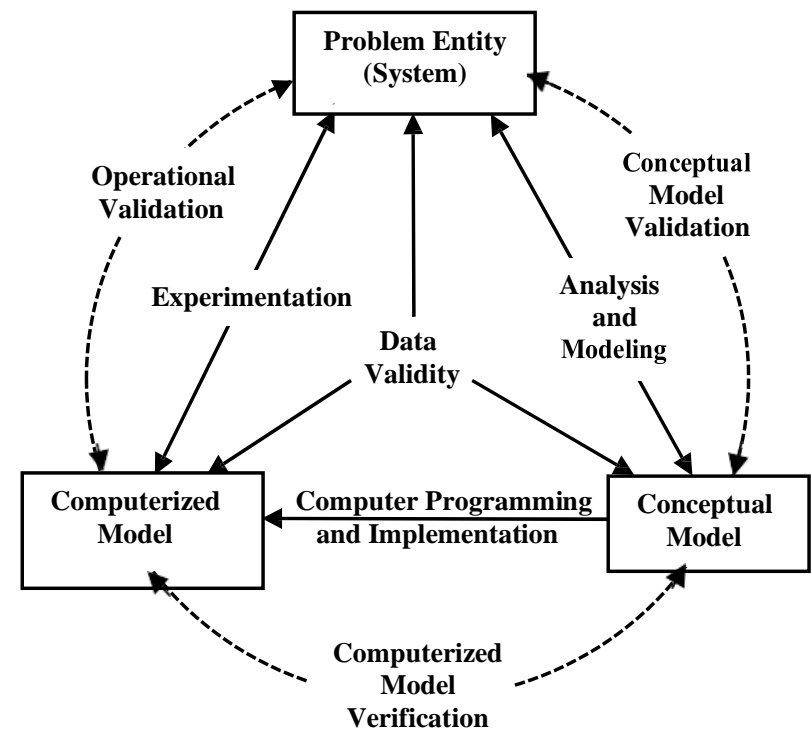

Figure 6: Model validation approach [13]

the chosen healthcare facility. We have secured ethics committee approval, and plan to run this trial as soon as final clinical post-discharge protocols are complete, validated, and approved and patient recruitment is complete.

For model validation, we adopt the approach described in [13]. Figure 6 illustrates the overall approach to model verification and validation. In this figure, Conceptual Model refers to a non-executable model notation whereas Computerized Model refers to a software implementation of such a model. However, in our case, a CPN model is both a conceptual model as well as a computerized model since CPN models 
themselves are executable. According to [13], model verification is the process of determining that a model implementation accurately represents the conceptual description and specifications whereas model validation is the process of determining the degree to which a model is an accurate representation of the real world. In particular, "...operational validation is carried out to determine the simulation model's output behavior has the accuracy required for the model's intended purpose over the domain of the model's intended applicability."

For data generation and validation purposes, CPN software provides an extensive monitoring and simulation report generation facility [14]. Simulation report provides a complete execution trace of the model whereas a monitor is a mechanism in the CPN software that is used to observe, inspect, control, or even modify a simulation of a CPN. A variety of monitors can be defined for a given net. Monitors can inspect both the markings of places and enabling of transitions during a simulation, and they can take appropriate actions based on the observations as well as extract relevant data. Figure 7 depicts a sample simulation report generated

\begin{tabular}{|ccl|}
\hline Step & Time & \multicolumn{1}{c|}{ Transition Occurence } \\
\hline 1 & 5 & Send_Activity_Data @ (1:Activity) \\
2 & 5 & Send_Weight_Data @ (1:Weight) \\
3 & 5 & Send_Pain_Data @ (1:Pain) \\
4 & 5 & Forward_To_HDAA @ (1:Home_Network) \\
5 & 5 & Add_To_PMR @ (1:HDAA_System) \\
6 & 5 & Forward_To_HDAA @ (1:Home_Network) \\
7 & 5 & Add_To_PMR @ (1:HDAA_System) \\
8 & 5 & Forward_To_HDAA @ (1:Home_Network) \\
9 & 5 & Add_To_PMR @ (1:HDAA_System) \\
10 & 5 & Send_BP_Data @ (1:Blood_Pressure) \\
11 & 5 & Forward_To_HDAA @ (1:Home_Network) \\
12 & 5 & Add_To_PMR @ (1:HDAA_System) \\
13 & 10 & Send_Pain_Data @ (1:Pain) \\
14 & 10 & Send_Activity_Data @ (1:Activity) \\
15 & 10 & Send_BP_Data @ (1:Blood_Pressure) \\
16 & 10 & Forward_To_HDAA @ (1:Home_Network) \\
17 & 10 & Add_To_PMR @ (1:HDAA_System) \\
18 & 10 & Forward_To_HDAA @ (1:Home_Network) \\
19 & 10 & Send_Weight_Data @ (1:Weight) \\
20 & 10 & Forward_To_HDAA @ (1:Home_Network) \\
21 & 10 & Add_To_PMR @ (1:HDAA_System) \\
22 & 10 & Forward_To_HDAA @ (1:Home_Network) \\
23 & 10 & Add_To_PMR @ (1:HDAA_System) \\
24 & 10 & Add_To_PMR @ (1:HDAA_System) \\
25 & 15 & Send_Hyd_Data @ (1:Hydration) \\
26 & 15 & Forward_To_HDAA @ (1:Home_Network) \\
$\ldots$ & $\ldots$ & ‥ \\
44 & 160 & Send_Pain_Data @ (1:Pain) \\
45 & 160 & Forward_To_HDAA @ (1:Home_Network) \\
46 & 160 & Add_To_PMR @ (1:HDAA_System) \\
47 & 162 & Send_Temp_Data @ (1:Body_Temp) \\
48 & 162 & Forward_To_HDAA @ (1:Home_Network) \\
49 & 162 & Add_To_PMR @ (1:HDAA_System) \\
50 & 182 & Send_Weight_Data @ (1:Weight) \\
51 & 182 & Forward_To_HDAA @ (1:Home_Network) \\
\hline
\end{tabular}

Figure 7: Sample simulation report generated by CPN software by the model under consideration for a sample run. Even in absence of any device data in the shown simulation report, the sequence of activities alone provide a good insight into the system behavior. Typically, such runs are useful in identifying any unsafe or undesirable behaviors. For situations where device and other data is crucial, the aforementioned monitoring facilities can be used to log necessary data during simulation.

\section{Conclusions}

This research in progress paper has served to proffer a solution to address the post discharge phase of stoma patients and thus begin to answer the posed research question. The proffered technology enabled solution supports both a high quality patient experience as well as a value-based care paradigm. We contend that such solutions are not just useful in the context as we have presented; i.e., stoma patients, but can be applied more generally so that the post dis-charge phase of the patient journey is also monitored and managed, enabling both a high quality patient experience as well as prudent management of likely trigger situations that may if not addressed lead to unplanned readmissions. Given the challenges faced by all OECD countries with respect to the exponential costs to provide quality care and the increasing pressures on healthcare organizations to deliver high quality and high value care we believe such technology solutions are strategic necessities and must be carefully considered. Hence, our study has significant and far reaching contributions to both theory and practice around enabling high value, high quality care to continue in the post discharge phase of the patient journey. Our future work will focus on conducting clinical trials to establish the validity, usability and proof of concept of the proffered solution.

\section{References}

[1] Australian Commission on Safety and Quality in Healthcare (2010) Patient-Centered Care: Improving Quality and Safety by Focusing Care on Patients and Consumers Discussion Paper. Department of Health and Ageing, Sydney.

[2] Kitson, A., Marshall, A., Bassett, K., \& Zeitz, K. (2013). What are the core elements of patient-centred care? A narrative review and synthesis of the literature from health policy, medicine and nursing. Journal of advanced nursing, 69(1), 4-15.

[3] Hibbard, J. H., \& Greene, J. (2013). What the evidence shows about patient activation: better health outcomes and care experiences; fewer data on costs. Health affairs, 32(2), 207-214. 
[4] Wildevuur, S. E., \& Simonse, L. W. (2015). Information and communication technology-enabled person-centered care for the "big five" chronic conditions: scoping review. Journal of Medical Internet Research, 17(3): e77. doi: 10.2196/jmir.3687

[5] Middleton, B., Bloomrosen, M., Dente, M. A., Hashmat, B., Koppel, R., Overhage, J. M., \& Zhang, J. (2013). Enhancing patient safety and quality of care by improving the usability of electronic health record systems: recommendations from AMIA. Journal of the American Medical Informatics Association: JAMIA, 20(e1): e2-e8. doi: 10.1136/amiajnl-2012-001458

[6] Collen, M. F. (2015). A history of medical informatics in the United States. M. J. Ball (Ed.). New York: Springer.

[7] Audet, A. M., Squires, D., \& Doty, M. M. (2014). Where are we on the diffusion curve? Trends and drivers of primary care physicians' use of health information technology. Health services research, 49(1 Pt 2): 347-360. doi: 10.1111/14756773.12139

[8] AIHW, 2017. Variation in hospital admission policies and practices: Australian hospital statistics Available at https://www.aihw.gov.au/reports/hospitals/variation-hospitaladmission-policies-practices/contents/table-of-contents accessed Sept 2018.

[9] Jensen, K \& Kristensen, L. M. (2009). Coloured Petri Nets: Modelling and Validation of Concurrent Systems, Springer-Verlag.

[10] Gehlot, V. \& Nigro, C. (2010). An Introduction to Systems Modeling and Simulation with Colored Petri Nets. Proceedings of the 2010 Winter Simulation Conference, pp. 104-118.

[11] Sloane, E. B. \& Gehlot, V. (2007). Use of Coloured Petri Net models in planning, design, and simulation of intelligent wireless medical device networks for safe and flexible hospital capacity management. International Journal of Networking and Virtual Organisations (IJNVO), Vol. 4, No. 2, pp. 118129.

[12] Reisig, W. (2013). Understanding Petri Nets. SpringerVerlag.

[13] Sargent, R. D. (2011). Verification and validation of simulation models. Proceedings of the 2011 Winter Simulation Conference, pp. 183-198.

[14] Lindstrøm, B., and L. Wells. (2002). Towards a Monitoring Framework for Discrete-Event System Simulations. In Proceedings of the Sixth International Workshop on Discrete Event Systems (WODES'02), pp. 127134. IEEE Computer Society.

[15] Bartels, S. J., Pratt, S.I., Aschbrenner, K. A., Barre, L. K., Jue, K., Wolfe, R. S., Xie, H., McHugo, G., Santos, M., Williams, G. E., Naslund, J. A., \& Mueser, K. T. (2013).
Clinically significant improved fitness and weight loss among overweight persons with serious mental illness. Psychiatr Serv. 64(8): 729-36. doi: 10.1176/appi.ps.003622012

[16] Michard, F., Mountford, W. K., Krukas, M. R., Ernst, F. R., \& Fogel, S. L. (2015). Potential return on investment for implementation of perioperative goal-directed fluid therapy in major surgery: a nationwide database study. Perioperative Medicine (London, England), 4, 11. doi:10.1186/s13741-0150021-0

[17] Michard, F., Giglio, M. T., \& Brienza, N. Perioperative goal-directed therapy with uncalibrated pulse contour methods: impact on fluid management and postoperative outcome. (2017). British Journal of Anaesthesia, 119 (1): 2230, doi: 10.1093/bja/aex138

[18] Pinsky, M. R., Clermont, G., \& Hravnak, M. (2016). Predicting cardiorespiratory instability. Critical Care, 20(1): 70-77. doi: 10.1186/s13054-016-1223-7

[19] Thacker, J, K. M., Mountford, W. K., Ernst, F. R., Krukas, M. R., \& Mythen, M. G. (2016). Perioperative Fluid Utilization Variability and Association With Outcomes: Considerations for Enhanced Recovery Efforts in Sample US Surgical Populations. Annals of Surgery, 263(3): 502-510. doi: 10.1097/SLA.0000000000001402

[20] Benes, J., Giglio, M., Brienza, N., \& Michard, F. (2014). The effects of goal-directed fluid therapy based on dynamic parameters on post-surgical outcome: a meta-analysis of randomized controlled trials. Critical Care, 18(5): 584-594. doi: 10.1186/s13054-014-0584-Z

[21] Taenzer, A. H., Pyke, J. B., McGrath, S. P., \& Blike, G. T. (2010). Impact of Pulse Oximetry Surveillance on Rescue Events and Intensive Care Unit Transfers: A Beforeand-After Concurrence Study. Anesthesiology 112(2):282287. doi: 10.1097/ALN.0b013e3181ca7a9b

[22] Brown, H., Terrence, J., Vasquez, P., Bates, D. W., \& Zimlichman, E. (2014). Continuous Monitoring in an Inpatient Medical-Surgical Unit: A Controlled Clinical Trial. The American Journal of Medicine, 127(3): 226-232. doi: 10.1016/j.amjmed.2013.12.004

[23] Subbe, C. P., Duller, B., \& Bellomo, R. (2017). Effect of an automated notification system for deteriorating ward patients on clinical outcomes. Critical Care, 21(1): 52-60. doi: 10.1186/s13054-017-1635-z

[24] Chen, G., Chung, E., Meng, L., Alexander, B., Vu, T., Rinehart, J., \& Cannesson, M. (212). Impact of non invasive and beat-to-beat arterial pressure monitoring on intraoperative hemodynamic management. Journal of Clinical Monitoring and Computing, 26(2): 133-140. doi: 10.1007/s10877-0129344-2

[25] Salmasi, V., Maheshwari, K., Yang, D., Mascha, E. J., Singh, A., Sessler, D. I., \& Kurz, A. (2017). Relationship between Intraoperative Hypotension, Defined by Either 
Reduction from Baseline or Absolute Thresholds, and Acute Kidney and Myocardial Injury after Noncardiac Surgery: A

Retrospective Cohort Analysis. Anesthesiology: The Journal of the American Society of Anesthesiologists, 126(1): 47-65. doi: 10.1097/ALN.0000000000001432 